\title{
ECHELLE MEASUREMENTS OF THE EXPANSION VELOCITIES OF THE FAINT GIANT HALOES OF PLANETARY NEBULAE
}

\author{
M.BRYCE ${ }^{1}$, J.MEABURN ${ }^{1}$ and J.R.WALSH ${ }^{2}$ \\ ${ }^{1}$ Department of Astronomy, University of Manchester \\ Oxford Road, Manchester M13 9PL. UK \\ ${ }^{2}$ Space Telescope European Coordinating Facility, ESO \\ Karl-Schwarzchild-Strasse 2, D-8046, Garching bei München, Germany
}

The faint, giant haloes observed in many Planetary Nebulae (PNe) are believed to be the remnants of the superwinds ejected by the progenitor asymptotic giant branch stars. Spatially resolved, high spectral resolution observations of [OIII] $5007 \AA$ line profiles from the haloes of NGC 6543 and NGC 6826 have been obtained with the Manchester echelle spectrometer at the Isaac Newton Telescope. The widths of the observed profiles from NGC 6826 decrease towards the outer edge of the halo which implies a definite radial expansion of this halo. The expansion velocities $v_{\text {exp }}$ of the two haloes are derived using both thick and thin shell models.

In the thin shell model, the halo is assumed to be a spherically expanding thin shell of constant density. The model predicts line splitting (not seen in either data set) if $v_{\text {exp }} \geq$ the component widths. This model halo is limb brightened. In the thick shell model, the halo is assumed to be filled with a radially expanding wind with density inversely proportional to the square of the radius. The resultant profiles are found to be $\sim$ Gaussian in shape. The surface brightness of this model halo falls off as $x^{-3}$ where $x$ is the angular distance from the centre of the nebula.

\begin{tabular}{|l|c|c|}
\hline Model & NGC 6543 & NGC 6826 \\
$v_{\text {exp }}$ Thin Shell $\left(\mathrm{kms}^{-1}\right)$ & $4.8 \pm 0.6$ & $9.8 \pm 0.6$ \\
$v_{e x p}$ Thick Shell $\left(\mathrm{kms}^{-1}\right)$ & $7 \pm 1$ & $13 \pm 1$ \\
\hline
\end{tabular}

\section{TABLE I}

Expansion velocities of the two haloes derived using thick and thin shell models.

The values of $v_{\text {exp }}$ derived for these haloes using the two models are shown in Table I. The thick shell model predicts a larger value of $v_{\text {exp }}$. The halo of NGC 6543 is filamentary and appears to be limb brightened. Data from the bright knot has been included in the calculations of $v_{\text {exp }}$, the results are not significantly affected if this data is omitted. Both predicted values of $v_{\text {exp }}$ for NGC 6543 are lower than the expected superwind expansion velocity which is $\sim 10 \mathrm{kms}^{-1}$. The halo of NGC 6826 is more uniform although somewhat mottled in appearance. Limb brightening at [OIII] $5007 \AA$ is not apparent, except for the bright knot. The decrease in observed profile width to larger angular radius indicates a systematic expansion of the halo. The value of $v_{\text {exp }}$ predicted by the thin shell model implies line splitting, which is not seen in the data. Together with the absence of limb brightening at [OIII] $5007 \AA$, this suggests that the thick shell model is more applicable to this halo than the thin shell model. 